\title{
Gametic disequilibrium and multilocus estimation of selfing rates
}

\author{
Philip W. Hedrick* and \\ Kermit Ritland $\dagger$
}

* Department of Biology, Pennsylvania State University, University Park, PA 16802, U.S.A. $\dagger$ Department of Botany, University of Toronto, Toronto, ON M5S 1A1, Canada.

The influence of disequilibrium between loci on multilocus estimates of the selfing (or outcrossing) rate has been examined. In general, there is a negative bias in estimating the outcrossing rate when there is disequilibrium but under certain conditions, there may be a small positive bias. When attempting to estimate the extent of biparental inbreeding using a comparison of the multilocus and single-locus estimates of outcrossing, the general negative bias caused by disequilibrium will result in an underdetection of biparental inbreeding.

\section{INTRODUCTION}

In recent years there has been an increased interest in mating systems, both because of the availability of biochemical marker loci to estimate components of the mating system and because of theoretical developments concerning the evolution of mating systems and related topics (e.g., Brown et al., 1985; Hedrick, 1990). When many polymorphic loci are available in a population, multilocus estimation of the selfing rate (or its complement, outcrossing) is appropriate (e.g., Brown et al., 1978; Green et al., 1980; Shaw et al., 1981; Ritland and Jain, 1981) because of its decreased statistical variance and increased robustness to violations of the mixedmating model. These techniques generally assume no statistical association, i.e., gametic equilibrium, between alleles at different loci in the pollen gametes. For unlinked loci in outbreeding organisms, this is generally true but in selfing organisms there is often gametic disequilibrium even between unlinked loci (e.g., Hedrick et al., 1978).

Shaw et al. (1981) used Monte Carlo simulation to examine the effects of gametic disequilibrium on their multilocus estimator. Summarizing their findings ( $t$ indicates the rate of outcrossing), they state that there is a "bias in estimation of $t$ (underestimation)..., with multilocus associations". However, the basis of this bias is not obvious and it is not clear under what conditions it is important. As a result, we will examine two procedures for estimation of the outcrossing rate when two loci are in disequilibrium, that of Shaw et al. (1981) and that of Ritland and Jain (1981), to understand why such a bias may occur.

\section{SHAW ET AL. MODEL}

First, let us reformulate the measure of Shaw et al. (1981) in the notation used by Ritland and Jain (1981). The expected estimate of the outcrossing rate is

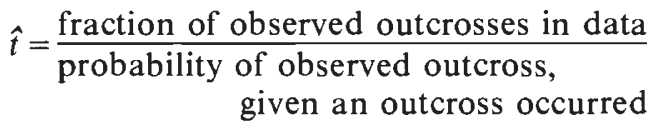

$$
\begin{aligned}
& =\frac{t \sum f_{i}\left(T_{i}+\Delta_{i}\right)}{\sum f_{i} T_{i}}
\end{aligned}
$$

where $f_{i}$ is the frequency of parent $i, T_{i}$ is the probability of observing outcross assuming no gametic disequilibrium $(D=0)$, and $\Delta_{i}$ is the deviation of $T_{i}$ due to $D$ (see table 1 for the values of these parameters for the nine two-locus genotypes). The bias in this estimate from the true outcrossing rate is

$$
\begin{aligned}
\hat{t}-t & =t\left(\frac{\sum f_{i} \Delta_{i}}{\sum f_{i} T_{i}}\right) \\
& =-t D\left(\frac{f_{1}-f_{3}-f_{7}+f_{9}}{\sum f_{i} T_{i}}\right) .
\end{aligned}
$$


Table 1 Probability of parental genotypes and of observing an outcross under Shaw et al. model

\begin{tabular}{|c|c|c|c|}
\hline \multicolumn{2}{|l|}{ Parental } & \multicolumn{2}{|c|}{$\begin{array}{l}\text { Probability of observing } \\
\text { an outcross, given an } \\
\text { outcross occurred }\end{array}$} \\
\hline Genotype & $\begin{array}{l}\text { Genotype } \\
\text { frequency } f_{\mathrm{i}}\end{array}$ & $\begin{array}{l}\text { Assuming } \\
D=0 \\
T_{\mathrm{i}}\end{array}$ & $\begin{array}{l}\text { Deviation } \\
\text { due to } D \\
\Delta_{\mathrm{i}}\end{array}$ \\
\hline $\mathrm{AABB}$ & $f_{1}$ & $1-p_{\mathrm{A}} p_{\mathrm{B}}$ & $-\mathrm{D}$ \\
\hline $\mathrm{AABb}$ & $f_{2}$ & $1-p_{\mathrm{A}}$ & 0 \\
\hline $\mathrm{AAbb}$ & $f_{3}$ & $1-p_{\mathrm{A}} p_{\mathrm{b}}$ & D \\
\hline $\mathrm{AaBB}$ & $f_{4}$ & $1-p_{\mathrm{B}}$ & 0 \\
\hline $\mathrm{AaBb}$ & $f_{5}$ & 0 & 0 \\
\hline Aabb & $f_{6}$ & $1-p_{\mathrm{b}}$ & 0 \\
\hline $\mathrm{aaBB}$ & $f_{7}$ & $1-p_{\mathrm{a}} p_{\mathrm{B}}$ & D \\
\hline $\mathrm{aaBb}$ & $f_{8}$ & $1-p_{a}$ & 0 \\
\hline aabb & $f_{9}$ & $1-p_{\mathrm{a}} p_{\mathrm{b}}$ & $-\mathrm{D}$ \\
\hline
\end{tabular}

This formula is independent of any assumptions about parental frequencies, and emphasizes that the proportions of parental genotypes used in estimating $t$ is important in determining bias.

Let us assume there is a high amount of selfing so that only double homozygous maternal plants are present in frequencies $f_{1}=p_{\mathrm{A}} p_{\mathrm{B}}+D, f_{3}=$ $p_{\mathrm{A}} p_{\mathrm{b}}-D, \quad f_{7}=p_{\mathrm{a}} p_{\mathrm{B}}-D$, and $f_{9}=p_{\mathrm{a}} p_{\mathrm{b}}+D$, for genotypes homozygous for gametes $\mathrm{AB}, \mathrm{Ab}, \mathrm{aB}$, and $a b$, respectively (see table 1 ). These are also assumed to be the frequencies of the four types of gametes in the pollen. Notice we have assumed here that there may be disequilibrium in both the egg and pollen gametes. In this case, the bias is

$$
\hat{t}-t=-D t\left(\begin{array}{c}
\frac{\left(p_{\mathrm{A}}-p_{\mathrm{a}}\right)\left(p_{\mathrm{B}}-p_{\mathrm{b}}\right)+4 D}{1-\left(p_{\mathrm{A}}^{2}+p_{\mathrm{a}}^{2}\right)\left(p_{\mathrm{B}}^{2}+p_{\mathrm{b}}^{2}\right)} \\
-D\left(p_{\mathrm{A}}-p_{\mathrm{a}}\right)\left(p_{\mathrm{B}}-p_{\mathrm{b}}\right)
\end{array}\right) \text {. }
$$

On the other hand, if we assume that the population is completely outbred and $p_{\mathrm{A}}=p_{\mathrm{B}}=0 \cdot 5$, then equation (2) given the bias is

$$
\hat{t}-t=\frac{-2 D^{2} t}{\frac{7}{16}-D^{2}} \text {. }
$$

Note that $D^{2} \leqq \frac{1}{16}$ so this bias is always negative.

Let us examine the bias in outcrossing rate as a function of the outcrossing rate and the extent of disequilibrium (see fig. 1). It is useful in this case to use the normalized measure of disequilibrium of Lewontin (1964) which is

$$
D^{\prime}=\frac{D}{D_{\max }}
$$

where $D_{\max }$ is the lesser of $p_{\mathrm{A}} p_{\mathrm{B}}$ or $p_{\mathrm{a}} p_{\mathrm{b}}$ if $D<0$ and $D_{\max }$ is the lesser of $p_{\mathrm{A}} p_{\mathrm{b}}$ or $p_{\mathrm{a}} p_{\mathrm{B}}$ if $D>0$. To
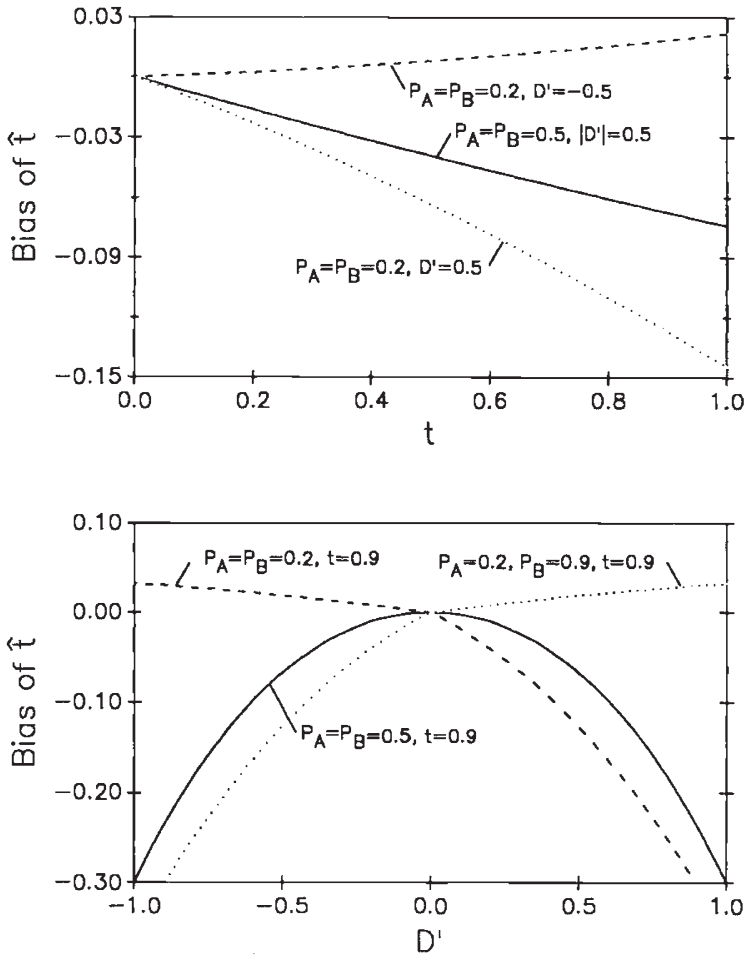

Figure 1 The bias in the outcrossing estimate as a function of the outcrossing rate and $D^{\prime}$ for several numerical combinations under the Shaw et al. model.

calculate the bias, the parental proportions were assumed to be

$$
\begin{aligned}
& f_{1}=(1-F) x_{\mathrm{AB}}^{2}+F x_{\mathrm{AB}} \\
& f_{2}=2(1-F) x_{\mathrm{AB}} x_{\mathrm{ab}} \\
& f_{3}=(1-F) x_{\mathrm{Ab}}^{2}+F x_{\mathrm{Ab}} \\
& f_{4}=2(1-F) x_{\mathrm{AB}} x_{\mathrm{aB}} \\
& f_{5}=2(1-F)\left(x_{\mathrm{Ab}} x_{\mathrm{aB}}+x_{\mathrm{AB}} x_{\mathrm{ab}}\right) \\
& f_{6}=2(1-F) x_{\mathrm{Ab}} x_{\mathrm{ab}} \\
& f_{7}=(1-F) x_{\mathrm{aB}}^{2}+F x_{\mathrm{aB}} \\
& f_{8}=2(1-F) x_{\mathrm{aB}} x_{\mathrm{ab}} \\
& f_{9}=(1-F) x_{\mathrm{ab}}^{2}+F x_{\mathrm{ab}}
\end{aligned}
$$

where $x_{\mathrm{AB}}, x_{\mathrm{Ab}}, x_{\mathrm{aB}}$, and $x_{\mathrm{ab}}$ are the frequencies of the gametes indicated by the subscripts and where the two-locus inbreeding coefficient is

$$
F=\frac{1-t}{1+t} .
$$

Notice in the top part of fig. 1 that the bias increases with $t$ and is generally negative. An 
example illustrating that the bias may be positive is also given $\left(p_{\mathrm{A}}=p_{\mathrm{B}}=0 \cdot 2, D^{\prime}=-0 \cdot 5\right)$ although the positive bias in this case is still small even for high $t$ values. When the bias is plotted as a function of the disequilibrium $D^{\prime}$ (bottom part of fig. 1), the bias is least when $D^{\prime}$ is near zero. However, if the disequilibrium is not close to zero, the bias can be quite negative in a number of situations or slightly positive in some other cases.

\section{RITLAND AND JAIN MODEL}

Ritland and Jain (1981) developed a procedure for the estimation of the outcrossing rate (and allelic frequencies) based on a multilocus maximum likelihood equation. They assumed that the loci were statistically independent (no gametic disequilibrium), an assumption that we relax here. The Ritland and Jain model differs from the Shaw et al. model primarily in that the Ritland and Jain model uses information about $t$ among heterozygous parents and also estimates the pollen pool from multilocus formulae.

The likelihood equation that specifies the probability of each parent-progeny observation is generally written as

$$
L=\prod_{\mathrm{i}} \prod_{\mathrm{j}}\left(t T_{\mathrm{ij}}+s S_{\mathrm{ij}}\right) P_{\mathrm{ij}}
$$

where $T_{\mathrm{ij}}$ is the frequency of progeny genotype $\mathrm{j}$ given parent genotype i outcrossed (see table 2), $S_{\mathrm{ij}}$ is the probability of progeny genotype $\mathrm{j}$ given parent genotype i selfed (see table 2), $s=1-t$, and $P_{\mathrm{ij}}$ is the expected proportion of the sample which has parent $\mathrm{i}$ and progeny $\mathrm{j}\left(\sum_{\mathrm{ij}} P_{\mathrm{ij}}=1\right)$. Note that here the $S_{\mathrm{ij}}$ and $T_{\mathrm{ij}}$ values assume independence of the two loci and that the $P_{\mathrm{ij}}$ values include disequilibria.

The maximum likelihood estimate of $t$ satisfies

$$
\frac{\mathrm{d} \ln L}{\mathrm{~d} t}=0=\sum_{\mathrm{i}} \sum_{\mathrm{j}} P_{\mathrm{ij}}\left(\frac{T_{\mathrm{ij}}-S_{\mathrm{ij}}}{\hat{s} S_{\mathrm{ij}}+\hat{t} T_{\mathrm{ij}}}\right) .
$$

If we multiply the above by $\hat{s} \hat{t}$ and add $\hat{t}$ to both sides, we obtain

$$
\hat{t}=\sum_{\mathrm{i}} \sum_{\mathrm{j}} f_{\mathrm{i}} P_{\mathrm{ij}}\left(\frac{\hat{t} T_{\mathrm{ij}}}{\hat{s} S_{\mathrm{ij}}+\hat{t} T_{\mathrm{ij}}}\right) .
$$

This expression can be used as an expectationmaximization (EM) recursion equation to solve for $\hat{t}$ (Ritland, 1986). Suppose the $P_{\mathrm{ij}}$ values are affected by linkage so that

$$
P_{\mathrm{ij}}=f_{\mathrm{i}}\left[s\left(S_{\mathrm{ij}}+\varepsilon_{\mathrm{ij}}\right)+t\left(T_{\mathrm{ij}}+\Delta_{\mathrm{ij}}\right)\right]
$$

where $f_{\mathrm{i}}$ is the frequency of parent $\mathrm{i}, \varepsilon_{\mathrm{ij}}$ is the deviation of selfed progeny from the expectation of independence when the loci are linked and is a function of the crossover rate $c$, and $\Delta_{i j}$ is the deviation of two-locus pollen allelic frequencies from exception of independence and is a function

Table 2 Values of $\mathrm{T}_{\mathrm{ij}}$ and $\mathrm{S}_{\mathrm{ij}}$ for each parent-progeny combination. It is assumed that coupling and repulsion genotypes are equal

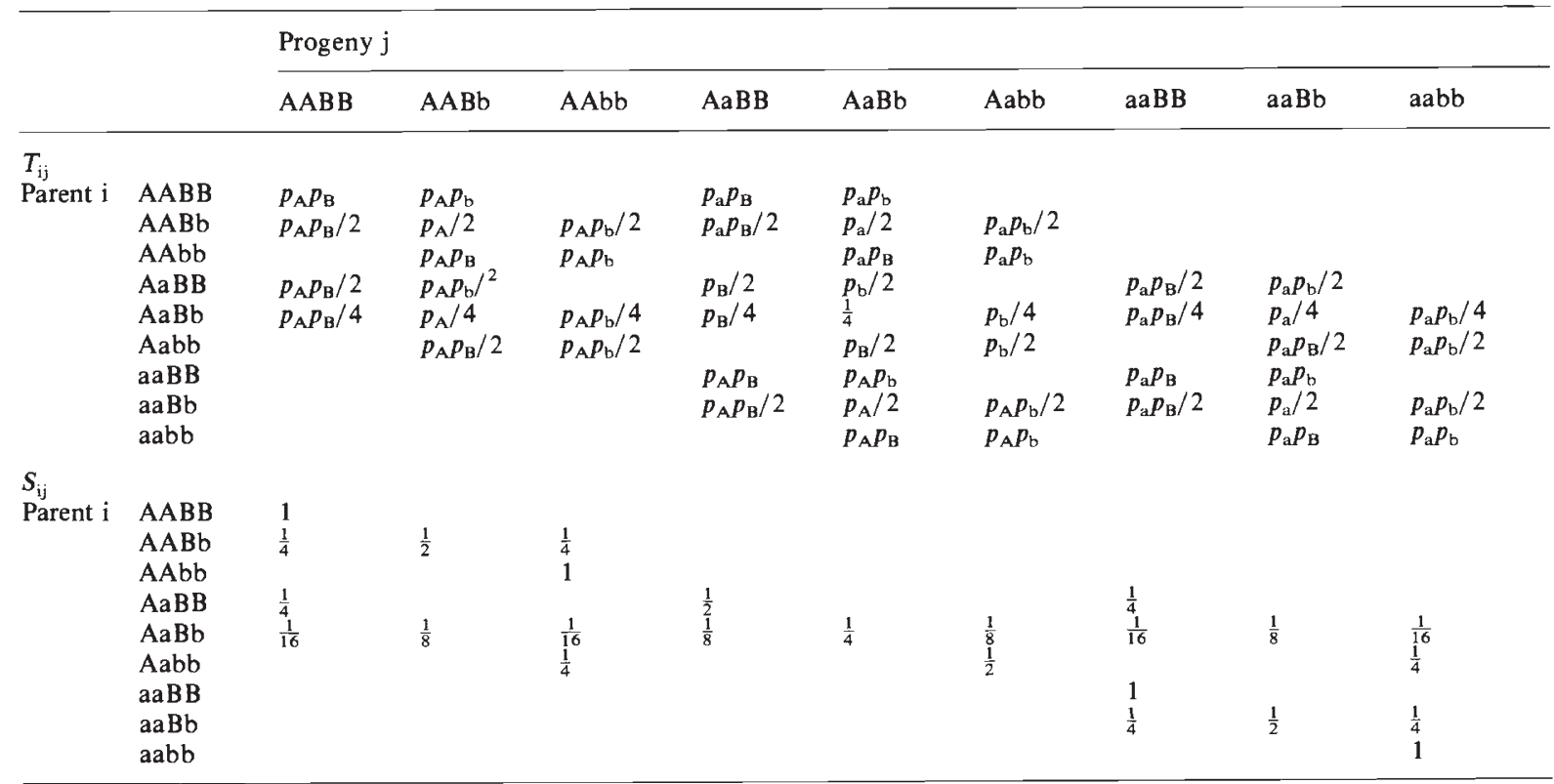


Table 3 Values of $\Delta_{\mathrm{ij}}$ and $\varepsilon_{\mathrm{ij}}$ for each parent-progeny combination where $e=1-4 c(1-c)$

\begin{tabular}{|c|c|c|c|c|c|c|c|c|c|c|}
\hline & & \multicolumn{9}{|c|}{ Progeny j } \\
\hline & & $\mathrm{AABB}$ & $\mathrm{AABb}$ & $\mathrm{AAbb}$ & $\mathrm{AaBB}$ & $\mathrm{AaBb}$ & Aabb & aaBB & $\mathrm{aaBb}$ & aabb \\
\hline $\begin{array}{l}\Delta_{\mathrm{ij}} \\
\text { Parent i }\end{array}$ & $\begin{array}{l}\text { AABB } \\
\text { AABb } \\
\text { AAbb } \\
\text { AaBB } \\
\text { AaBb } \\
\text { Aabb } \\
\text { aaBB } \\
\text { aaBb } \\
\text { aabb }\end{array}$ & $\begin{array}{l}L \\
D / 2 \\
\\
D / 2 \\
D / 4\end{array}$ & $\begin{array}{l}-D \\
D \\
-D / 2 \\
D / 2\end{array}$ & $\begin{array}{l}-D / 2 \\
-D \\
-D / 4 \\
-D / 2\end{array}$ & $\begin{array}{l}-D \\
-D / 2\end{array}$ & $\begin{array}{l}D \\
-D\end{array}$ & $\begin{array}{l}D / 2 \\
D\end{array}$ & $\begin{array}{l}-D / 2 \\
-D / 4 \\
-D \\
-D / 2\end{array}$ & $\begin{array}{l}D / 2 \\
D / 2 \\
D \\
-D\end{array}$ & $\begin{array}{l}D / 4 \\
-D / 2 \\
\\
D / 2 \\
D\end{array}$ \\
\hline $\begin{array}{l}\varepsilon_{\mathrm{ij}} \\
\text { Parent i }\end{array}$ & $\begin{array}{l}\text { not } \mathrm{AaBb} \\
\mathrm{AaBb}\end{array}$ & $\begin{array}{l}0 \\
\frac{e}{16}\end{array}$ & $\begin{array}{l}0 \\
-\frac{e}{8}\end{array}$ & $\begin{array}{l}0 \\
\frac{e}{16}\end{array}$ & $\begin{array}{l}0 \\
-\frac{e}{8}\end{array}$ & $\begin{array}{l}0 \\
\frac{e}{4}\end{array}$ & $\begin{array}{l}0 \\
-\frac{e}{8}\end{array}$ & $\begin{array}{l}0 \\
\frac{e}{16}\end{array}$ & $\begin{array}{l}0 \\
-\frac{e}{8}\end{array}$ & $\begin{array}{l}0 \\
\frac{e}{16}\end{array}$ \\
\hline
\end{tabular}

of $D$ (the values of $\varepsilon_{\mathrm{ij}}$ and $\Delta_{\mathrm{ij}}$ are given in table 3 ; note $\varepsilon_{\mathrm{ij}}$ is needed only for double heterozygotes). Because the Ritland and Jain model uses information from all parents, the crossover rate (in addition to $D$ ) may affect $\hat{t}$ because selfed progeny of double heterozygotes may differ in genotypic frequency according to $c$. The bias is obtained by substitution of expression (10) into (9) and then subtracting $t$ which gives

$$
\begin{aligned}
\hat{t}-t= & \sum_{\mathrm{i}} f_{\mathrm{i}} \sum_{\mathrm{j}}\left[s\left(S_{\mathrm{ij}}+\varepsilon_{\mathrm{ij}}\right)\right. \\
& \left.+t\left(T_{\mathrm{ij}}+\Delta_{\mathrm{ij}}\right)\right]\left(\frac{\hat{t} T_{\mathrm{ij}}}{\hat{s} S_{\mathrm{ij}}+\hat{t} T_{\mathrm{ij}}}\right)-t .
\end{aligned}
$$

This equation is an implicit function of $\hat{t}$ so an analyical expression like (2) is not possible.

The bias for the Ritland and Jain model is plotted in fig. 2 where (11) was solved numerically using the bisection method, for the same values as fig. 1 , assuming that the recombination frequency is 0.5 among double-heterozygous parents that self (i.e., $\varepsilon_{\mathrm{ij}}=0$ and $c=\frac{1}{2}$ in table 3 ). At $p=0.5$, there is little information about $t$ among heterozygous parents, so bias is practically the same for the two models. At other allelic frequencies, the bias is less for the Ritland and Jain model. In the parameter combinations which gave a positive bias for the Shaw et al. model, the amount of positive bias when it occurred was much less for the Ritland and Jain model. If $c=0$ (no recombination), the bias is changed by at most 5 per cent of its value. This is again because double-heterozygous parents (the only parents that need recombination to be specified supply little information about $t$.

\section{DISCUSSION}

The statement by Shaw et al. (1981) that there is a negative bias in estimating $t$ when there are
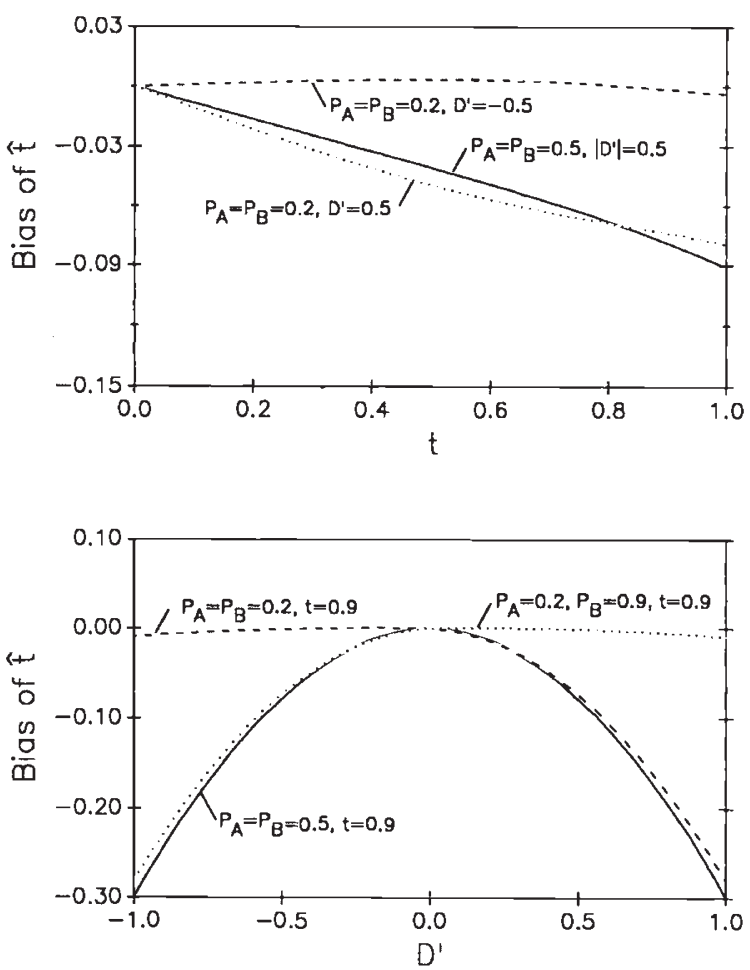

Figure 2 The bias in the outcrossing estimate as a function of the outcrossing rate and $D^{\prime}$ for several numerical combinations under the Ritland and Jain model. 
multilocus associations appears to be generally true for both the estimator they propose and the estimator of Ritland and Jain (1981). However, under certain combinations of allelic frequencies and gametic disequilibrium, there can be a small positive bias. Both the extent of bias and the possibility of positive bias values are larger for the Shaw et al. model than for the Ritland and Jain model. We emphasize that we have computed bias for the two-locus case; inclusion of more loci would generally reduce the biases computed here. However, it is very difficult to find the expected biases with three or more loci because of both the complexity of the parameter space (three $D$ values are needed for three loci) and the necessity for higher-order disequilibria to completely describe the cases of three or more loci.

How can we intuitively understand the basis of this bias? An increase in disequilibrium tends to decrease gametic diversity and thus increase the probability that uniting two-locus gametes are identical in state. The estimator equates this increased identity in state with an increased identity by descent (which is caused by selfing) and hence overestimate selfing. This effect can be quantified by examining the frequency of double homozygotes in the presence of disequilibrium. The excess of this frequency over that expected with no disequilibrium is

$$
\begin{aligned}
\left(p_{\mathrm{A}} p_{\mathrm{B}}+D\right)^{2}+\left(p_{\mathrm{A}} p_{\mathrm{b}}-D\right)^{2}+\left(p_{\mathrm{a}} p_{\mathrm{B}}-D\right)^{2} \\
\quad+\left(p_{\mathrm{a}} p_{\mathrm{b}}+D\right)^{2}-p_{\mathrm{A}}^{2} p_{\mathrm{B}}^{2}-p_{\mathrm{A}}^{2} p_{\mathrm{b}}^{2}-p_{\mathrm{a}}^{2} p_{\mathrm{B}}^{2}-p_{\mathrm{a}}^{2} p_{\mathrm{b}}^{2} \\
=D\left(p_{\mathrm{A}}-p_{\mathrm{a}}\right)\left(p_{\mathrm{B}}-p_{\mathrm{b}}\right)+4 D^{2} .
\end{aligned}
$$

This excess must be positive for selfing to be overestimated, i.e., for a negative bias of $\hat{t}$ to occur, or in other words, if

$$
D^{2}>\frac{1}{4} D\left(p_{\mathrm{A}}-p_{\mathrm{a}}\right)\left(p_{\mathrm{B}}-p_{\mathrm{b}}\right)
$$

is true, a negative bias of $\hat{t}$ occurs (otherwise the bias is positive). Considering specific cases, if either locus has intermediate allelic frequencies $\left(p_{\mathrm{A}}=p_{\mathrm{a}}\right.$ or $\left.p_{\mathrm{B}}=p_{\mathrm{b}}\right)$, then the bias of $\hat{t}$ will always be negative, and indeed figs 1 and 2 show this. If $p_{\mathrm{A}}$ and $p_{\mathrm{B}}$ are both greater than 0.5 or both less than $0 \cdot 5$, the bias of $\hat{t}$ will be negative when $D>0$ but positive when $D<0$. Conversely, if $p_{\mathrm{A}}$ and $p_{\mathrm{B}}$ lie on opposite sides of $0 \cdot 5$, the bias of $\hat{t}$ will be positive when $D>0$ and negative when $D<0$. Both of these qualitative conclusions are borne out by figs 1 and 2 .
If we define $D$ as the covariance of the most common alleles at each locus, the general rule that emerges is that positive $D$ causes a downward bias of $\hat{t}$ and negative $D$ causes a slight upward bias of $\hat{t}$. This general downward bias rule is relevant for studies that attempt to detect biparental inbreeding by comparing single-locus estimates of $t\left(t_{\mathrm{s}}\right)$ with multilocus estimates of $t\left(t_{\mathrm{m}}\right)$. Shaw et al. (1981) and subsequent workers have noted that the difference $t_{\mathrm{m}}-t_{\mathrm{s}}$ is a rough indicator of the proportion of selfing caused by biparental inbreeding. If $t_{\mathrm{m}}$ is biased downward by gametic disequilibrium $D$ and since $t_{\mathrm{s}}$ is unaffected by $D$, biparental inbreeding will be underestimated or perhaps not even detected.

We stress that the bias of $t$ also depends upon the parental genotypic proportions, as shown by (2). Conceivably, this bias can be eliminated by weighting parental proportions, or more interestingly, estimating $t$ for each parental genotype. However, other problems emerge when $t$ is estimated for individual genotypes (Ritland and Hedrick, in preparation).

\section{REFERENCES}

BROWN, A. H. D., BARRETT, S. C. H. AND MORAN, G. F. 1985. Mating system estimation in forest trees: models, methods, and meaning, p. 32-49. In Gregorius, H.-R. (ed.) Population Genetics in Forestry, Springer, Berlin.

BROWN, A. H. D., ZOHARY, D. AND NEVO, E. 1978. Outcrossing rates and heterozygosity in natural populations of Hordeum spontaneum Koch in Israel. Heredity, 41, 49-62.

GREEN, A. G., BROWN, A. H. D. AND ORAM, R. N. 1980. Determination of outcrossing rate in a breeding population of Lupinus albus L. (white lupin). Z. Pflanzenzüchtg, 84, 181191.

HEDRICK, P. W. 1990. The mating system and evolutionary genetics. In Wöhrmann, K. and Jain, S. (eds) Population Biology: Ecological and Evolutionary Viewpoints, SpringerVerlag, Berlin.

HEDRICK, P. W., JAIN, S. AND HOLDEN, L. 1978. Multilocus systems in evolution. Evol. Biol., 11, 101-182.

LEWONTIN, R. C. 1964. The interaction of selection and linkage. I. General considerations; heterotic models. Genetics, 49 , 49-67.

RITLAND, K., AND JAIN, S. 1981. A model for the estimation of outcrossing rate and gene frequencies fusing $\mathrm{n}$ independent loci. Heredity, 47, 35-52.

RITLAND, K. 1986. Joint maximum likelihood estimation of genetic and mating structure using open-pollinated progenies. Biometrics, 42, 25-43.

SHAW, D. V., KAHLER, A. L. AND ALlard, R. W. 1981. A multilocus estimation of mating system parameters in plant populations. Proc. Natl Acad. Sci. USA, 78, 1298-1302. 